\title{
Study on Environment Friendly Descaling and Formation Unblocking Technique for Injection Wells
}

\author{
Yan yu, Yang yang, Guo shuzhao, Cai qingqin, Li qinghua \\ Petroleum Engineering Research Institute of Dagang Oilfield, Tianjin 300280
}

\begin{abstract}
Corrosion and scale formation of near well-bore zone are common reasons that affect the effectiveness of injection wells. Although conventional stimulation treatments can remove the blockage, many disadvantages occur such as short-term effectiveness, long occupation period, large amount of waste fluid, big environmental impact and limited transportation procurement. In this paper, an environment friendly descaling and formation unblocking technique was found which includes two kinds of treatments. One is called neutral descaling, anti-swelling, liquid circulation cleaning technique(NALC) and the other is called slow release anti-scaling solid acid treatment(SASA). The environment impacts of well stimulation treatments are significantly reduced which would meet the requirements of the "Green Mine" needs.
\end{abstract}

\section{Introduction}

Usually, the use of formation energy to extract oil is called primary oil recovery; water and gas injection to supplement formation energy is called secondary oil recovery; chemicals and hot fluid injection to change the viscosity and other physical figures is called third oil recovery ${ }^{[1-2]}$. With the continuous development of the "second and third recovery enhancement" program of Dagang Oilfield, the number of injection wells grows rapidly and injection networks are continuously improved. Due to the water quality, compatibility, temperature and pressure changes of the injection fluid, scaling and plugging occurs in the near well-bore zone, which may increase the injection pressure and lower the injection volume ${ }^{[3-4]}$. Nearly $30 \%$ injection wells in the studied area were affected. The former has carried out physical cleaning, ultrasonic cleaning, chemical dissolution and other descaling and plugging process for the injection wells whose tubing extends to two or three kilometers and more. Although these treatments may reduce the water injection pressure, it has disadvantages such as strong tubing corrosion, easy to generate hydro sulfide, short effective period and easy sand burying. For acid-sensitive formations, the use of acidification treatments has a multiple risk. Meanwhile, as "Green Mine" program was promoted, more regions set a higher level of requirements for "environment friendly procedure". Especially for environment sensitive areas, the demand for cleaning operations is increasing due to the limitation of acid procurement and transportation requirements.

\section{Scale type analysis of injection wells}

Injecting well scaling is known as the process that equipment interfaces and formation interval are gradually covered by scale pollutant. The causes of common scaling of oilfield injection wells are shown in Figure 1. According to the scaling causes analysis, physical condition changes of the formation are considered the mainly causes of scaling in the near well-bore zone and the inner tube ${ }^{[5]}$. For example, high salinity and high oxygen content of injecting fluid, incompatible working fluid with reservoir water and so on.

According to more than 200 times indoor experiments, the scaling of the on-site equipment is mainly caused by corrosion. The main component is $\mathrm{Fe} 2+$ and $\mathrm{Fe} 3+$, which may cause $\mathrm{H} 2 \mathrm{~S}$ pollution. The main components of the near-well scale are $\mathrm{Ca} 2+, \mathrm{Ba} 2+$ and other cationic compound. The scale type is mainly carbonate, and some injection wells contain sulfate.

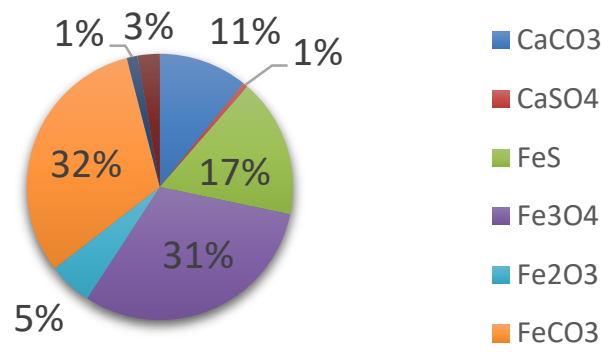

Figure 1 Scale components of the injection wells

\section{Environment friendly descaling and unblocking fluid system}

\subsection{Neutral descaling, anti-swelling liquid}

Since the scale in the injection well usually has iron-ion compounds, organic matter, gravel, clay mixture, the working fluid is required to have the characteristics of sequestration, anti-swelling, penetrating, dispersing and 
stabilizing, so as to quickly penetrate the scale, enlarge the chelation reaction area, and stabilize the reservoir particles $^{[6 \sim 7]}$.

The hydrochloric acid solution has a big dissolve rate for carbonate scale, but for sulfate and silicate scale it is less than $20 \%$. The macro cyclic polyether is the main components of the Neutral descaling, anti-swelling fluid. It causes lattice distortion through the adsorption of crystallites in the scale which increases the inter molecular repulsive force. Also it forms a stable soluble chelate with $\mathrm{Ca} 2+, \mathrm{Mg} 2+, \mathrm{Ba} 2+$ and other cations in the well and then dissolute scale ${ }^{[8]}$. The $30 \%$ of the neutral descaling and unblocking agent solution may significantly increase the rate of dissolution of calcium sulfate scale, barium sulfate scale and silicate scale by $54 \%, 38 \%$ and $51 \%$, respectively. Also, because there are no free hydrogen ions in the liquid, it wills minimum the corrosion of the well bore and the column, meanwhile; avoid the risk to generate hydrogen sulfide. It can also be applied to the descaling and unblocking treatments in acid-sensitive formations.

Table 1 Comparison of dissolving ability of hydrochloric acid and the neutral descaling solution

\begin{tabular}{|c|c|c|c|c|}
\hline Scale type & $\mathrm{CaCO} 3$ & $\mathrm{CaSO} 4$ & $\mathrm{BaSO} 4$ & $\mathrm{CaSiO} 3$ \\
\hline $15 \% \mathrm{HCl}, \%$ & 95 & 17 & 9 & 12 \\
\hline The neutral descaling solution, $\%$ & 25 & 71 & 47 & 63 \\
\hline
\end{tabular}

Table 2 Comparison of corrosion rate of hydrochloric acid and the neutral descaling solution

\begin{tabular}{|c|c|c|c|}
\hline Fluid system & Temperature, ${ }^{\circ} \mathrm{C}$ & Time, $\mathrm{h}$ & Corrosion Rate, $\mathrm{g} /\left(\mathrm{m}^{2} \cdot \mathrm{h}\right)$ \\
\hline 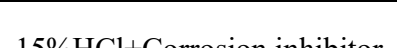 & \multirow{4}{*}{90} & \multirow{4}{*}{4} & 1.365 \\
\hline $15 \% \mathrm{HCl}+\mathrm{Corrosion}$ innibitor & & & 1.387 \\
\hline \multirow{2}{*}{ The neutral descaling solution } & & & 0.216 \\
\hline & & & 0.211 \\
\hline
\end{tabular}

The solution liquid used for descaling and unblocking of the injection well will directly enter the formation. In order to prevent the hydration and expansion of clay mineral, which will lead blockage of seepage channel and increase of injection pressure. The anti-swelling agent must be added to Reduces the damage ${ }^{[9]}$. The purpose of the anti-swelling agent evaluation experiment is based on the analysis of the core water sensitivity test, according to SY/T 5762 "Clay Stabilizer Performance Test Method for Fracturing and Acidizing treatments" and Q/SY DG 1190 "General Testing Method for Clay Stabilizer for Water Injection Wells". Experiments and tests were carried out to evaluate the effects of the anti-swelling agents. The results are shown in Table 3.

Table 3 Anti-swelling agent evaluation

\begin{tabular}{|c|c|c|c|c|c|}
\hline No. & 1 & 2 & 3 & 4 & 5 \\
\hline System Type & ZCY-07 & ZCS-05 & WD-5 & A-26 & FS-1 \\
\hline Anti-swelling rate, \% & 92.8 & 93 & 92 & 86.7 & 89.4 \\
\hline Cumulative flush, ml & 4500 & 4300 & 4400 & 4200 & 3900 \\
\hline Permeability damage rate, \% & 2.1 & 0.8 & 4.8 & 2.5 & 16 \\
\hline
\end{tabular}

Considering the anti-swelling rate, effective period, reservoir damage and other aspects, ZCS-05 has strong performance and is suitable for the neutral descaling solution.

For the mixed scaling in the near-well zone with calcium carbonate, calcium sulfate, barium sulfate and silicate scale, it is difficult to obtain a good descaling and unblocking fluid by using a single unblocking liquid. As one kind of acidizing additives, the anti-swelling agent used with a low-concentration acid to treat the carbonate scale, at the same time, utilizes the dissolution ability of the neutral descaling liquid to remove the blockage by sulfate scale.

\subsection{Slow releasing solid acid system}

Several sampling results showed that there was a big difference of water quality between water injection station and ending injection pipeline. An amount of suspended solids and inorganic impurities were found by the very end of the injection pipeline. Acid wash may remove the impurities, but the disadvantages are also obvious: high cost of treatments, long working period and especially for the injection of wells, the difficulty to operate acidizing treatments is further increased ${ }^{[10]}$. 


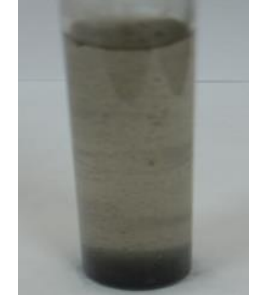

water sample

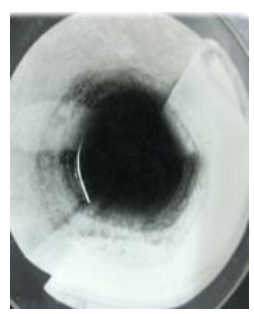

filtration

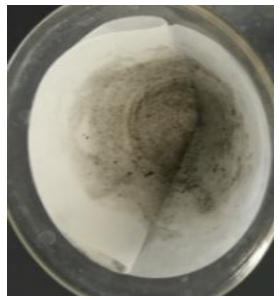

acid dissolution
Figure 2: Wellhead water sample acid dissolution

The slow releasing solid acids have obvious application advantages in manner of safety and environmental requirements. It is composed of water-soluble gel, slow-release skeleton, main acid, corrosion inhibitor. It has a small solubility to the formation rocks, thus, it has may increase the porosity of the formation. Meanwhile, due to the weak acidity, the dissolution of the formation rocks is limited, so it will not lead well bore collapse. The release rate of the slow releasing solid acid is related to the injection liquid salinity, reservoir temperature, flow velocity, and fluid contact area.

The release rate of the slow releasing solid acid was determined at $60^{\circ} \mathrm{C}$ under a flow rate of $0.1 \mathrm{~L} / \mathrm{min}$ by measuring the cumulative acid content of the liquid flowing out over a period of time. The dissolution time of the slow release scale solid acid decreases with increasing temperature and water flow rate. Experiment results showed that the acid release rate increased with the increase of temperature. At $60^{\circ} \mathrm{C}$, the average release rate of active ingredients of Slow releasing solid acid in 10 days was $21.6 \mathrm{~g} / \mathrm{d}$.

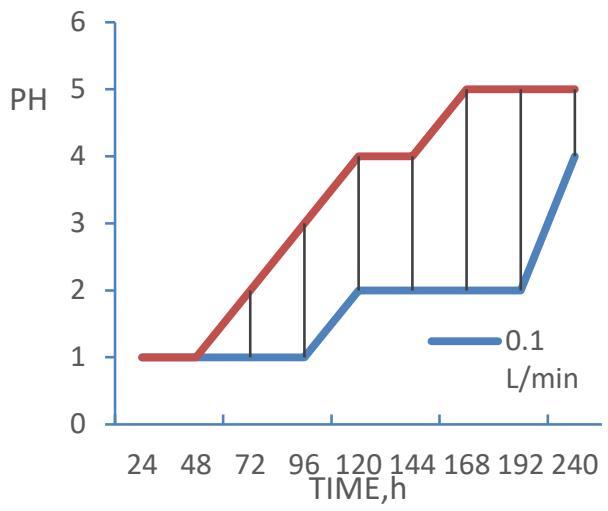

Figure $3 \mathrm{pH}$ profile of Slow releasing solid acid over time

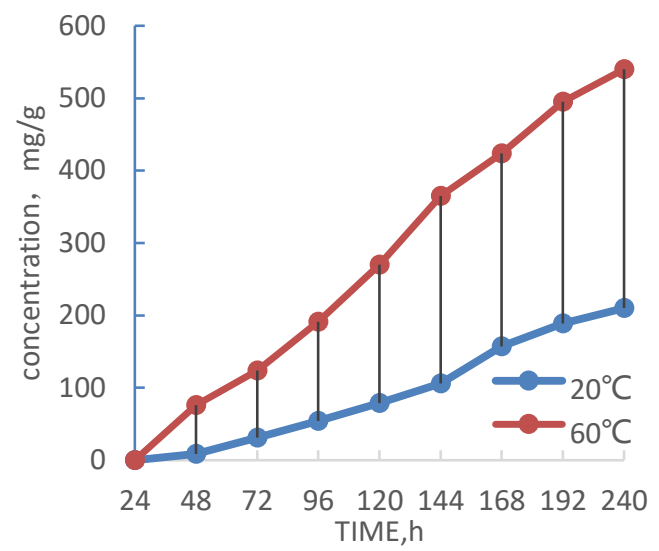

Figure4 Curve of acid concentration of Slow releasing solid acid with time

\section{Studies of environment friendly descaling and unblocking technique}

On one side, well strings will pull out to protect the equipment (such as packer and concentric water distributor) before conventional descaling treatment which will increase the cost and time ${ }^{[11]}$. On the other side, well killing fluid will cause formation damage as well as a large amount of waste (It will produce $40 \mathrm{~m} 3$ waste fluid for the well depth of $3000 \mathrm{~m}$ on a 2 times wash).

Environment friendly descaling and unblocking technique has low environmental impact, no waste fluid, and low energy consumption operation mode. The treatment process should be selected according to the type of scale of the target well and the purpose of operation.

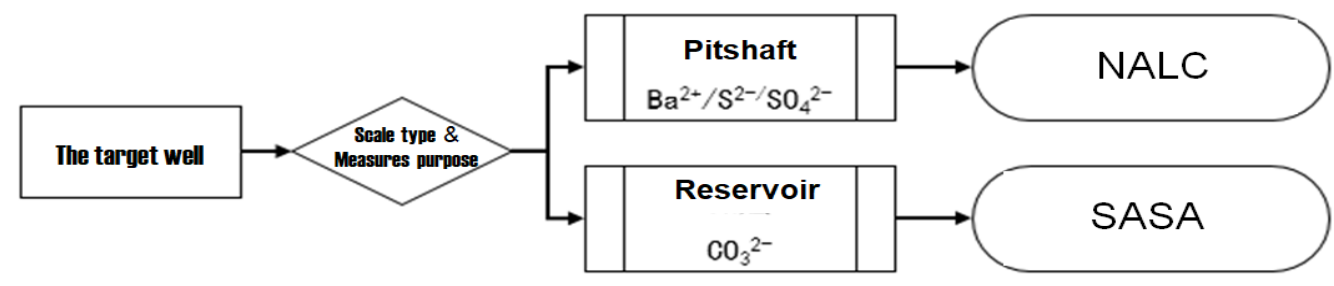

Figure 5: Cleaning descaling and plugging process selection method 


\subsection{Neutral descaling, anti-swelling, liquid circulation cleaning technique(NALC)}

Neutral descaling, anti-swelling, liquid circulation cleaning technique(NALC) is a process of a fixed pipe column without expensive fracturing trucks. It is only necessary to connect the tubing and casing valve then use the well washing vehicle to replaced neutral descaling and anti-swelling liquid is into the well bore. (According to Q/SY 111 "Oilfield chemical agent, drilling fluid biological toxicity classification and detection method, luminescent bacteria method"). After 24 hours of soaking, start circulation and filtrate the fluid 2 times of the well bore volume. The circulation will be completed after the indicators are displayed normally. The working fluid does not need to be replaced. After the injection line is restored, the working fluid will enter the reservoir and clean the near well bore zone.

\subsection{Slow release anti-scaling solid acid treatment(SASA)}

In order to extend water injection period and reduce the high cost, risk and operation complexity, the slow releasing solid acid is under discussion. It is pressed into a cylinder with a diameter of $30-55 \mathrm{~mm}$, and is put into the well bore during the water injection or in the outer joint which outside the pipe. There is no need to stop the injection in the descaling operation. Also there is no need of extra power equipment. The solid acid can be slowly released, so that the water penetrates into the formation reach a certain acidity, so as to dissolve the scale in the near well bore and slower scale forming.

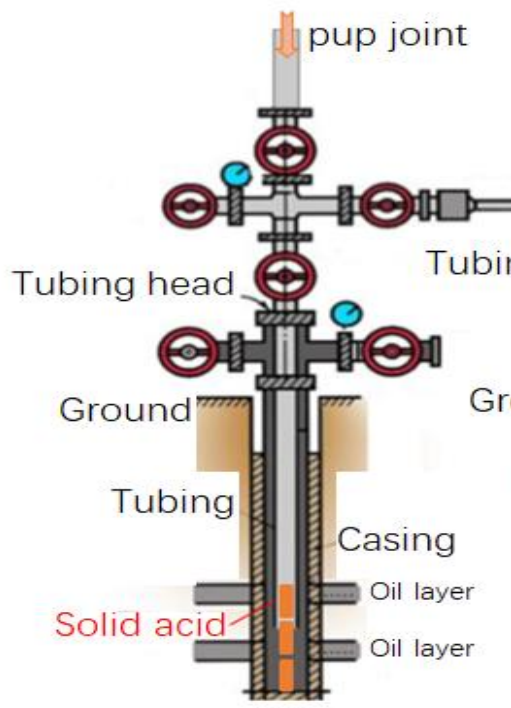

(1)

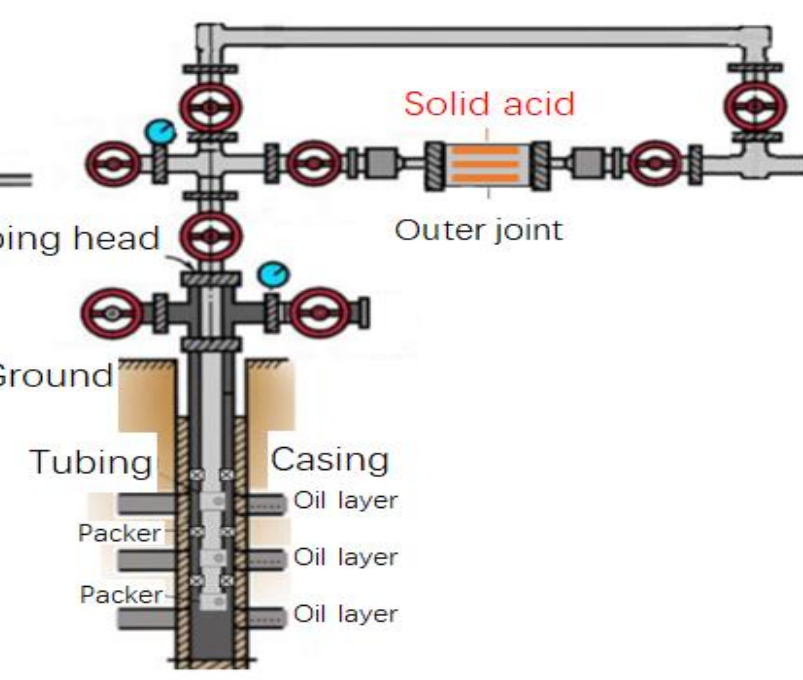

(2)

Figure 6 Diagram of slow releasing solid acid implementing mode

\subsection{On site application}

H121 is an injection well in the target block. After water injection in November 2018, the injection pressure increased rapidly from $11 \mathrm{MPa}$ to $21 \mathrm{MPa}$ within three months. The initial daily injection was $40 \mathrm{~m} 3$. The water injection pressure increased as the injection was carried on. The injection amount was lowered to $30 \mathrm{~m} 3$ from Jan.
2019. In April, Slow releasing solid acid online descaling technique was carried out. 60 columns of solid acid which were $18 \mathrm{~cm}$ long each were put into the basket of the pipe. After two times solid acid descaling treatment the injection pressure dropped from $15 \mathrm{MPa}$ to $12 \mathrm{MPa}$, and the injection volume continued to be stable which means solid acid descaling treatment is remarkable. 


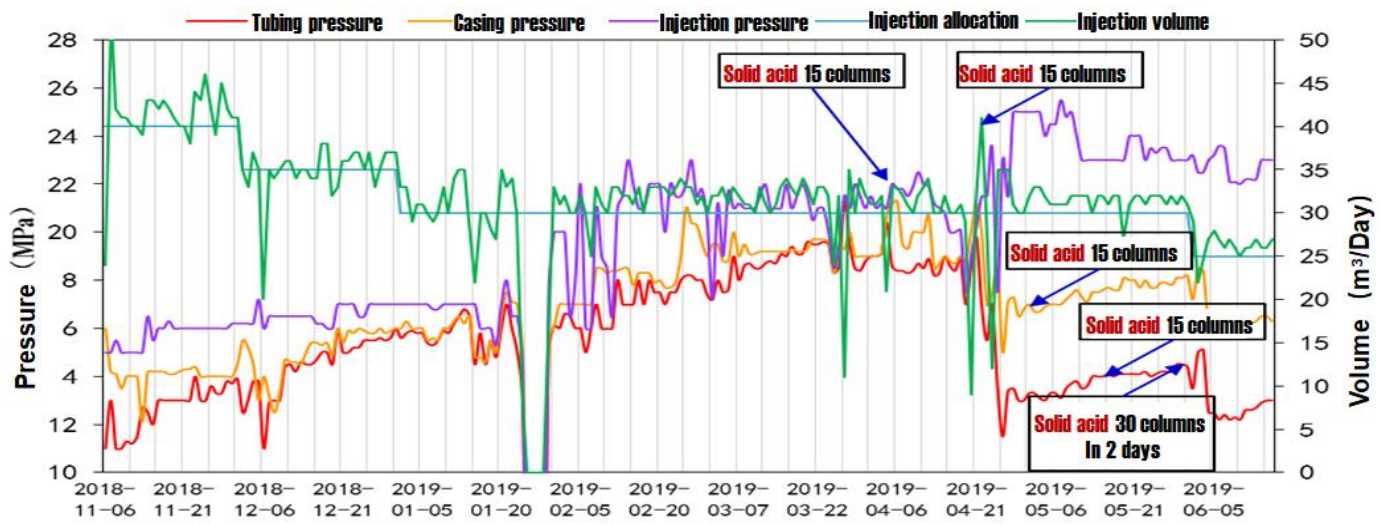

Figure 7 Daily Injection curve of well H121

\section{Conclusion}

Advantages of environment friendly descaling and unblocking technique for injection wells are as follows: (1) The consumption of power to implement descaling treatment was lower by more than $60 \%$. (2) It reduces the waste liquid by more than $80 \%$ compared with the traditional descaling process. (3) Save time for more than 12 hours per operation.

The wetlands, reservoirs and tidal flats in the Dagang Oilfield area are interlaced with the wellsite, thus requirements related to environment are complex and important. The treatments carried out in environmentally sensitive areas should be carefully carried out. The acid liquid transportation and the treatment of waste liquid after descaling treatments are all facing the challenges of safety and environmental protection. The study and practice of environment friendly descaling and unblocking technique for injection wells is of great significance to the "green mines" program promoted by Dagang Oilfield.

\section{References:}

1. Chang Samuel Hsu,Paul R. Robinson. Production for Recovery[M].Springer International Publishing:2019, 97-125.

2. He Jiang-chuan etc. Study on the Relationship Between Secondary Development and Tertiary Recovery Technology[J]. Journal of Southwest Petroleum Institute, 2011, 33(3): 96-100.

3. Zhang liying. Polymer injection well deepening and flushing technique $[\mathrm{J}]$. Oil-Gasfield Surface Engineering,2014,5,96.

4. Guan gongshuai etc. Plug Removal Effect of Compound Plug Removal Agent in Alkali-surfactant-polymer Flooding Injection Wells of Daqing Oilfield[J]. Oilfield Chemistry ,2016,33(3):442-444,455.

5. Qiu yinan. Reservoir evaluation technology[M]. Beijing: Petroleum Industry Press,1997,306-310.
6. Zhang weihua. A brief Analysis of the Corrosion Prevention Technology of Oil Field Pipeline[J]. Total Corrosion Control, 2018,32(9):85-86.

7. Yu cunye. Chelating Washing Technology for Difficult Fouling on Petrochemical Equipment and Application[J]. Corrosion \& Protection in Petrochemical Industry, ,2002,19(2):52-56.

8. Xu huashan, Ren yufen etc. Comparison of static scale-inhibiting properties of typic organo-phosphonate [J]. Chinese Journal of Environmental Engineering, 2007,2,6-10.

9. Qian sheng etc. New Method for Evaluating the Performance of Clay-swelling Stabilizer[J]. Journal of Oil and Gas Technology, 2010,32(5):129-131.

10. Yang jianhua. Scale buildup analysis and prevention technique in waterflood development oilfield[J]. Petroleum Geology and Recovery Efficiency, 2001,8(4):55-56.

11. Bing shaoxian,Li zhixue etc. Comparative analysis of production cost structure of oil field at home and abroad [J]. Journal of China University of Petroleum(Edition of Social Sciences),2008,5,5-9. 